\title{
Transition Metal Ions in Semiconductors: LDA, LDA+U, and Experiment
}

\author{
T. ZAKRZEWSKI ${ }^{a, *}$ AND P. BogusŁAWsKI ${ }^{a, b}$ \\ ${ }^{a}$ Institute of Physics, Polish Academy of Sciences, al. Lotników 32/46, 02-668 Warszawa, Poland \\ ${ }^{b}$ Faculty of Physics, Kazimierz Wielki University, pl. J. Weyssenhoffa 11, 85-072 Bydgoszcz, Poland \\ Electronic structure of $\mathrm{Cr}, \mathrm{Mn}, \mathrm{Fe}$, and Co transition metal (TM) ions in GaN and AlN was calculated \\ within generalized gradient approximation and GGA $+U$. The $+U$ term was considered as a free parameter with \\ $0<U<5 \mathrm{eV}$. Comparison with available data for intracenter optical transitions for Fe and Mn shows that good \\ agreement is obtained for values of $U$ smaller than $1 \mathrm{eV}$.
}

DOI: $10.12693 /$ APhysPolA.127.321

PACS: 71.15.Mb, 71.55.-i, 71.55.Eq, 75.50.Pp

\section{Introduction}

The known failure of the local density approximation (LDA) or generalized gradient approximation (GGA) is the underestimation of the band gap in solids, ascribed to the oversimplified treatment of exchange-correlation effects. The underestimation is particularly drastic in transition metal (TM) oxides. A considerable improvement is obtained by adding the $+U$ correction for particular atomic orbitals $[1,2]$. While the impact of $+U$ terms was extensively discussed for ideal crystals, its impact on the electronic structure of defects is less understood. However, apart from the practical aspects, this issue is of fundamental importance because, as it is shown here, it provides a demanding test for the schemes improving LDA or GGA, complementing the simple requirement of the correct band gap. Infact, centres such $\mathrm{Mn}$ and $\mathrm{Fe}$ ions in $\mathrm{GaN}$ are typical examples of defects that induce a few levels in the gap, and their intracenter transitions are known with high accuracy. We find that the energies of the defect levels are more sensitive to the $U$ corrections than the band gap itself. An aspect important for theory is that the $U$-corrected level energies depend on the occupancy, i.e., on the defect charge state.

In this paper we analyse the impact of the $+U$ term for $\mathrm{Cr}, \mathrm{Mn}, \mathrm{Fe}$, and $\mathrm{Co}$ ions in GaN and AlN. The $+U$ term was treated as a free parameter, and it was applied to $p(\mathrm{~N})$ and $d(\mathrm{TM})$ orbitals. The results of $\mathrm{GGA}+U$ calculations were compared to available experimental data. The $+U$ terms strongly affect the electronic structure of TM impurities. Surprisingly, for $U(\mathrm{TM})=0$, the energies of the gap levels induced by these centres, and of the intra-centre optical transitions, agree well with experiment. In contrast, for $U(\mathrm{~N})=U(\mathrm{TM})=5 \mathrm{eV}$, these energies are in substantial disagreement with experimental values by about $1-2 \mathrm{eV}$.

* corresponding author; e-mail: tzakrz@ifpan.edu.pl

\section{Method of calculations}

We performed GGA calculations with the PerdewBurke-Ernzerhof (PBE) exchange-correlation potential [3], using the $+U$ corrections implemented in the QUANTUM-ESPRESSO code [4] according to Ref. [1]. We employed ultrasoft atomic pseudopotentials, the wurtzite $(w)$ 128-atom supercell, the plane wave basis with the kinetic energy cutoff of 40 Ry. The Brillouin zone summations were performed using the MonkhorstPack scheme with a $2 \times 2 \times 2 k$-point mesh [5], and the Methfessel-Paxton smearing width of $0.068 \mathrm{eV}$. Ionic positions were optimized until the forces acting on ions were smaller than $0.02 \mathrm{eV} / \AA$.

The $+U$ correction was imposed on $2 p[6,7]$, giving the band gap of GaN is $3.2 \mathrm{eV}$. This is because the main orbitals contributing to top of the valence band (TVB) are $2 p(\mathrm{~N})$. Adding the $+U$ term to $d(\mathrm{Ga})$ opens the gap

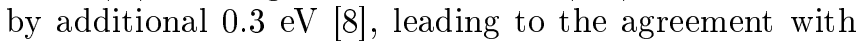
the experimental value $3.5 \mathrm{eV}$. The $+U$ correction for $\mathrm{TM}$ ions was considered as a free parameter varying from 0 to $5 \mathrm{eV}$.

\section{Results}

We consider here four common TM ions that substitute for Ga, in two wide band gap III-V semiconductors, GaN and AlN. In general, the atomic $d$-shell of a substitutional TM ion is split by two factors, namely the crystal field and the exchange interaction. First, the crystal field generated by the four tetrahedrally-coordinated nearest neighbors of TM splits the $d(\mathrm{TM})$ shell into an $e$ doublet and a $t_{2}$ triplet. The splitting is of the order of $1 \mathrm{eV}$, and the triplet higher in energy. Owing to the hexagonal symmetry of the wurtzite host and to the coupling with more distant neighbors, the $t_{2}$ further splits into a doublet and a singlet with a splitting energy of about $0.15 \mathrm{eV}$, which is small due to the smallness of the hexagonal perturbation. Second, the $d$-shell is split into spin-up and spin-down states by the exchange interaction of about 1-2 eV. The charge state of a TM ion depends on the 
position of the Fermi level $E_{\mathrm{F}}$, which in general is determined by the presence of intentional dopants, defects, etc. Here, we consider GaN that contains only the ana- lyzed TM ions, and thus their charge state is $3+$, which is assumed in the present calculations. The calculated energy levels of $\mathrm{Cr}, \mathrm{Mn}$, and Co are presented in Fig. 1.
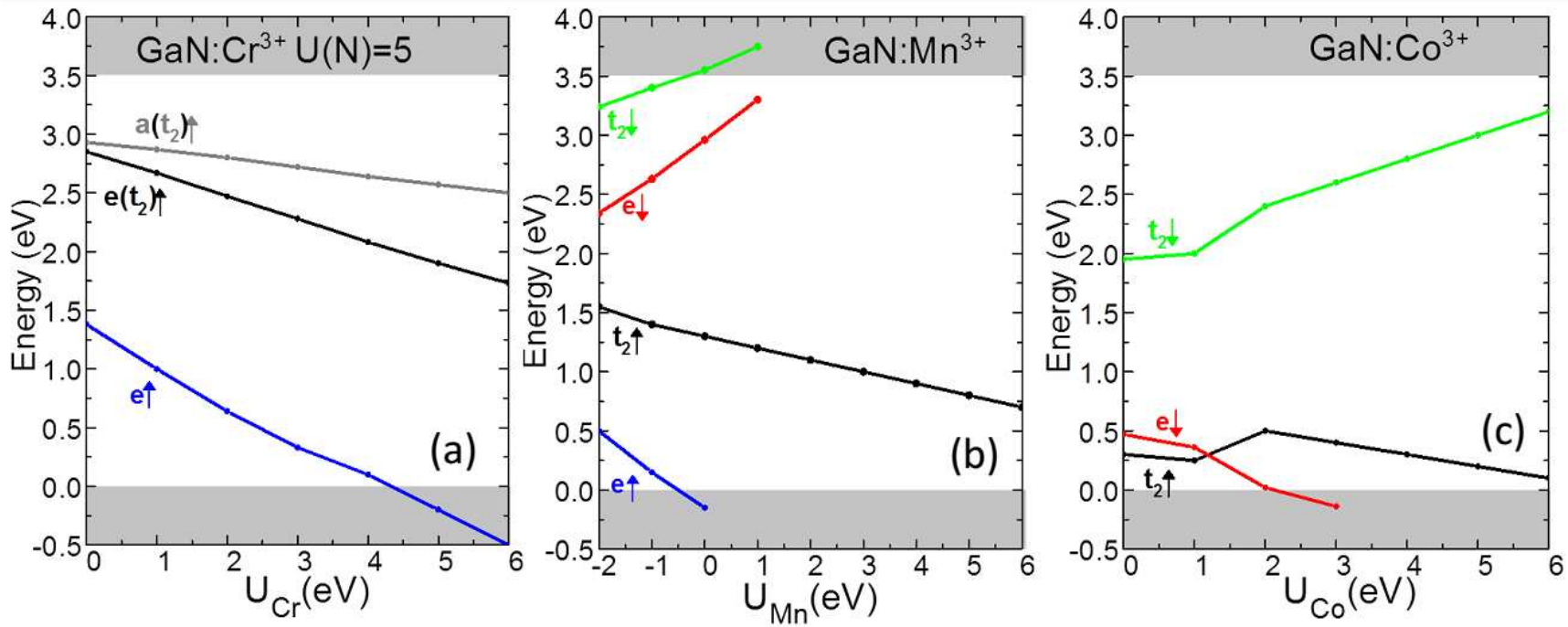

Fig. 1. Calculated energy levels of (a) $\mathrm{Cr}^{3+}$, (b) $\mathrm{Mn}^{3+}$, and (c) $\mathrm{Co}^{3+}$ ions in $\mathrm{GaN}$ as a function of $U(\mathrm{TM}) \cdot U(\mathrm{~N})=5 \mathrm{eV}$.
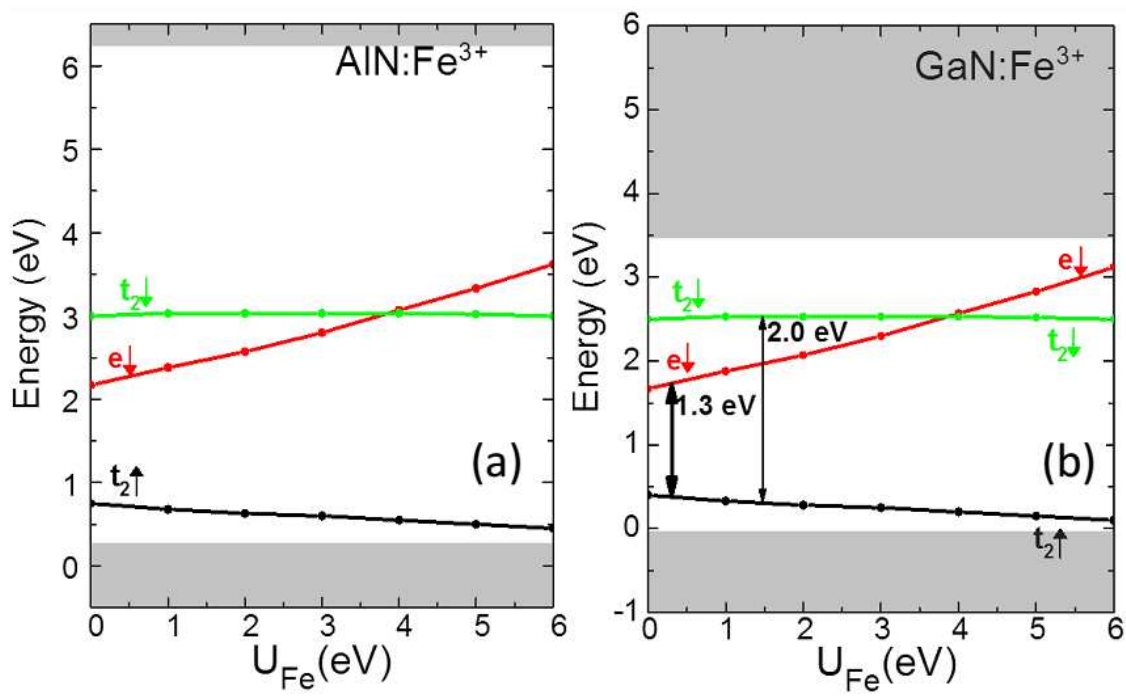

Fig. 2. Calculated energy levels of $\mathrm{Fe}^{3+}$ in (a) AlN and (b) GaN as a function of $U(\mathrm{Fe}) . U(\mathrm{~N})=0$.

As it follows from Fig. 1, the impurity levels strongly depend on the value of $U(\mathrm{TM})$. The dependence is explained by the fact that the $+U$ term is applied to a set of "localized" states $\{|m \sigma\rangle\}[1]$, which is our case are $p(\mathrm{~N})$ and $d(\mathrm{TM})$ orbitals with spin $\sigma$. The corresponding $U$-induced potential depends on occupations $n_{m \sigma}$, and is attractive (repulsive) for occupied (unoccupied) orbitals. If $|v \boldsymbol{k} \sigma\rangle$ denotes the band states, then

$$
n_{m \sigma}=\sum f(v \boldsymbol{k} \sigma)\langle v \boldsymbol{k} \sigma \mid m \sigma\rangle\langle m \sigma \mid v \boldsymbol{k} \sigma\rangle,
$$

where $f(v \boldsymbol{k} \sigma)$ is the occupation number. The induced energy correction is [2]:

$$
\Delta \varepsilon_{m \sigma}=U\left(1 / 2-n_{m \sigma}\right) .
$$

The $U$-induced energy shift of a given state $|v \boldsymbol{k} \sigma\rangle$ is determined by the contributions of $|m \sigma\rangle$ to this state, which can be found by appropriate projections. This contribution is in turn determined by hybridization between $\{|m \sigma\rangle\}$ and the host. If a defect state is mainly built up from $\{|m \sigma\rangle\}$, its energy is shifted by about $|U / 2|$. In the opposite limit, the state of an effective-mass acceptor (donor) is built from states from the valence (conduction) band extremum, and its energy relative to the top of the valence band (or bottom of the conduction band) are almost insensitive to $U$. 
$\mathrm{Cr}$ in $\mathrm{GaN}$ is in the $d^{3}$ configuration, with two electrons on the $e \uparrow$ level, and one on the $t_{2} \uparrow$. The hexagonal Jahn-Teller splitting of the latter state into $e\left(t_{2}\right)$ and $a\left(t_{2}\right)$ is enhanced by the occupation effect described by Eq. (2).

This splitting of $t_{2} \uparrow$ is much smaller for Mn, and therefore it is not shown in Fig. 1. However, in this case the spin-down states are in the gap, they are empty, and their upward energy shifts (see Fig. 1) with the increasing $U$ follow from Eq. (2). This argument also explains the downward shift of $e \downarrow$ of $\mathrm{Co}^{3+}$, Fig. 1c, which is occupied by one electron. In this case one can also see that $t_{2} \uparrow$ is strongly hybridized with the top of the valence band, and thus is less sensitive to $U$ than $e \downarrow$, which does not interact with the hole states. The degree of hybridization was directly found by analyzing the relevant wave functions.

The levels of $\mathrm{Fe}^{3+}$ in $\mathrm{AlN}$ and GaN are compared in Fig. 2. We see that the relative Fe level energies are very similar in both matrices. In other words, both the crystal field splitting and the exchange splitting of $\mathrm{Fe}$ ion levels weakly depend on the host, which is partially due to the similar ionicities and lattice constants of AlN and GaN. Moreover, the dependence of levels on $U(\mathrm{Fe})$ is almost the same.

We now compare the obtained results with experimental results, which allows to determine the optimal values of $U$. For $\mathrm{Fe}^{3+}$, two intracenter optical transitions were observed, at 1.3 and $2.01 \mathrm{eV}$. They are denoted by arrows in Fig. 2b. The $\mathrm{Fe}^{3+} /{ }^{2+}$ charge transfer level was assessed to be at $2.87 \mathrm{eV}[9]$. The best agreement with these data occurs for $U(\mathrm{Fe})=0$ and $U(\mathrm{~N})=0$. For $U(\mathrm{Fe})=U(\mathrm{~N})=5 \mathrm{eV}$ (and for hybrid functionals [1011]) the calculated exchange splitting of Fe states increases, and the error is as large as $2 \mathrm{eV}$. For $\mathrm{Mn}^{3+}$, intracenter absorption $e \uparrow \rightarrow t_{2} \uparrow$ at $1.4 \mathrm{eV}$ was identified [12-14], and $e_{2 \uparrow}$ was located $\approx 0.3 \mathrm{eV}$ above the TVB [12]. Ionization absorption $\mathrm{Mn}^{3+}+\mathrm{h} \nu \rightarrow \mathrm{Mn}^{2+}+$ hole at $1.8 \mathrm{eV}$ was also observed $[12,13]$. Comparison with the present results, Fig. 1b, shows that even $U(\mathrm{Mn})=0$ is too high a value, and all the above transitions are well reproduced by $U(\mathrm{~N})=0$ and a negative $U(\mathrm{Mn}) \approx-1.5 \mathrm{eV}$.

\section{Conclusions}

We have performed GGA and GGA $+U$ calculations of the energy levels of four typical TM impurities in GaN and AlN. The results were compared with experimental intracenter optical transition energies, which are known with high accuracies. The $+U$ term was considered as a free parameter. The strong dependence of the $U$ values of the crystal surrounding is demonstrated by the fact that the optimal values of $U$ are about $0-1 \mathrm{eV}$, i.e., they are smaller than those typically assumed for e.g. TM oxides, about $5 \mathrm{eV}$.

\section{Acknowledgments}

This work was supported by grant NCN 2012/05/B/ST3/03095.

\section{References}

[1] M. Cococcioni, S. de Gironcoli, Phys. Rev. B 71, 035105 (2005).

[2] V.I. Anisimov, F. Aryasetiawan, A.I. Lichtenstein, J. Phys. Condens. Matter 9, 767 (1997).

[3] J.P. Perdew, K. Burke, M. Ernzerhof, Phys. Rev. Lett. 77, 3865 (1996).

[4] http://www.pwscf.org.

[5] H.J. Monkhorst, J.D. Pack, Phys. Rev. B 13, 5188 (1976).

[6] I. Slipukhina, Ph. Mavropoulos, S. Blugel, M. Lezaic, Phys. Rev. Lett. 107, 137203 (2011).

[7] The $+U$ term was succesfully applied also to $p(\mathrm{O})$ orbitals, see R. Kovacik, C. Ederer, Phys. Rev. B 80, 140411(R) (2009).

[8] A. Janotti, D. Segev, C.G. Van de Walle, Phys. Rev. B 74, 045202 (2006).

[9] E. Malguth, A. Hoffmann, M.R. Phillips, Phys. Status Solidi B 245, 455 (2008).

[10] P. Alippi, F. Filippone, G. Mattioli, A. Amore Bonapasta, V. Fiorentini, Phys. Rev. B 84, 033201 (2011).

[11] A. Stroppa, G. Kresse, Phys. Rev. B 79, 201201 (2009).

[12] T. Graf, M. Gjukic, M.S. Brandt, M. Stutzmann, O. Ambacher, Appl. Phys. Lett. 81, 5159 (2002).

[13] A. Wolos, A. Wysmolek, M. Kaminska, A. Twardowski, M. Bockowski, I. Grzegory, S. Porowski, M. Potemski, Phys. Rev. B 70, 245202 (2004).

[14] R.Y. Korotkov, J.M. Gregie, B.W. Wessels, Appl. Phys. Lett. 80, 1731 (2002). 